\title{
Indirect exchange coupling between magnetic adatoms in carbon nanotubes
}

\author{
A. T. Costa, Jr.* \\ Departamento de Ciências Exatas, Universidade Federal de Lavras, 37200-000 Lavras, MG, Brazil \\ D. F. Kirwan and M. S. Ferreira ${ }^{\dagger}$ \\ Physics Department, Trinity College Dublin, Dublin 2, Ireland \\ (Received 23 March 2005; published 1 August 2005)
}

\begin{abstract}
The long-range character of the exchange coupling between localized magnetic moments indirectly mediated by the conduction electrons of metallic hosts can play a significant role in determining the magnetic order of low-dimensional structures. Here we consider how this indirect coupling influences the magnetic alignment of adatoms attached to the walls of carbon nanotubes. A general expression for the indirect coupling in terms of single-particle Green functions is presented. Contrary to the general property that magnetic moments embedded in a metal display Friedel-like oscillations in their magnetic response, calculated values for the coupling across metallic zigzag nanotubes show monotonic behavior as a function of the adatom separation. Rather than an intrinsic property, the monotonicity is shown to reflect a commensurability effect in which the coupling oscillates with periods that coincide with the lattice parameter of the nanotube host. Such a commensurability effect does not dominate the coupling across semiconducting zigzag or metallic armchair nanotubes. We argue that such a long-range character in the magnetic interaction can be used in future spintronic devices.
\end{abstract}

DOI: 10.1103/PhysRevB.72.085402

PACS number(s): 75.75.+a, 73.63.Fg

\section{INTRODUCTION}

More than a decade after the discovery of carbon nanotubes, these nanoscale cylindrical structures are still the subject of intensive scientific research due to their intriguing physical properties. Significant progress has been made to explain the intrinsic properties of nanotubes but in order to expand the applicability of those systems we need to understand how they are affected by the interaction with other objects. Nanotubes interacting with magnetic foreign objects are now in focus due to the possibility of implementing the technologically promising area of spintronics in molecular structures. In fact, the ability to produce sizeable changes in the conductance of a nanotube due to an applied magnetic field is one of the driving forces in the research of magnetic properties of carbon-based structures. ${ }^{1}$ The existence of such a spin-valve effect has potential applications in the development of faster, smaller, and more efficient nanoscopic magnetoelectronic devices.

Due to their inherent spin asymmetry, the interaction with magnetic foreign objects is likely to cause a spin-dependent response on the transport properties of the combined structure. Substrates, ${ }^{2,3}$ substitutional impurities, ${ }^{4}$ adsorbed atoms, ${ }^{5,6}$ and nanoparticles ${ }^{7}$ are some of the different magnetic foreign objects that can interact with carbon nanotubes. Among those, transition-metal magnetic adatoms have been reported to produce noticeable changes in the spin-dependent electronic structure of carbon nanotubes. ${ }^{5,7}$

In addition to establishing how magnetic impurities affect the electronic structure of the nanotube, it is crucial to understand the nature of the coupling between nearby adatoms since the transport properties of a magnetically doped structure depend on how the impurity moments are oriented. Dipolar and exchange interactions are the basic mechanisms defining the alignment of the adatoms. ${ }^{8}$ The former decays rather quickly as the moments are moved apart whereas the latter depends on both the dimensionality and on the nature of the interaction. Direct exchange coupling results from the overlap between wave functions centered at the magnetic impurities but also decays abruptly as the distance between the impurities increases. Of indirect nature, the exchange interaction between magnetic impurities mediated by the conduction electrons of the non-magnetic host is known to decay more slowly and plays an important role in determining the overall magnetic alignment of the system.

The coupling between magnetic moments mediated by the conduction electrons of nonmagnetic materials has been widely studied, both theoretically and experimentally. The main focus of recent work has been on metallic multilayers, ${ }^{9}$ where magnetic slabs are separated by layers of nonmagnetic metals. These systems are typically tridimensional, with translational symmetry preserved in two of the three dimensions. Many of the features of the exchange coupling in these systems are directly linked to their geometrical properties and therefore are expected to change appreciably for systems of lower dimensionality such as carbon nanotubes. In particular, it has been predicted that the indirect exchange coupling through a one-dimensional electron gas is long ranged. ${ }^{10}$ If this prediction holds for the coupling of magnetic adatoms mediated by nanotubes, this long-ranged magnetic interaction could lead to a degree of correlation in the electronic potential of magnetically doped nanotubes. From the perspective of fundamental science, such a spindependent correlated disorder is certain to affect the magnetotransport response of these structures, justifying the interest of our study. On the applied side on the other hand, our study is justified by the fact that detailed knowledge and, perhaps, control of this magnetic coupling opens the possibility of building quantum logic gates in solid state environments, reminding us of the huge potential of these structures as future components of spintronic devices. 


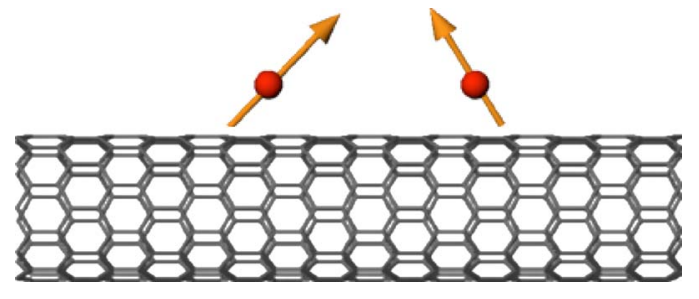

FIG. 1. (Color online) Schematic representation of the magnetic adatoms on a carbon nanotube.

With this motivation, in this article we investigate the indirect magnetic coupling between two magnetic adatoms attached to the walls of a carbon nanotube. By studying the energetic balance of the system as a function of the relative angle between the atomic magnetizations we can estimate the nature and magnitude of the coupling across the nanotube. In this way, we can obtain not only how the magnetic moments are aligned but also how it decays as the adatoms are moved further apart. The sequence adopted in this article is the following. We start by deriving a closed-form expression describing the energy required to rotate the relative magnetizations of the two adatoms. Since this expression involves matrix elements of the single-particle Green function of the system, it is worth presenting in the subsequent section a general way of evaluating these matrix elements for achiral carbon nanotubes. Results and discussions are presented in Sec. IV, followed by conclusions.

\section{INDIRECT MAGNETIC COUPLING}

We consider two magnetic atoms, labeled $A$ and $B$, adsorbed onto the walls of an infinitely long carbon nanotube and schematically represented in Fig. 1. Magnetism in these atoms is driven by an intra-atomic Coulomb interaction that, when treated in mean-field approximation through a selfconsistent procedure, can be described by an effective spindependent potential located at the atomic positions. In this way, the electronic structure of the entire system is well described by a single-particle Hamiltonian in a basis of localized atomic orbitals. In such a basis, the tight-binding-like Hamiltonian is fully determined by the on-site potentials and hopping integrals.

We start by assuming that the magnetic moments of the individual adatoms are initially parallel, hereafter referred to as the ferromagnetic (FM) configuration. In this configuration, the Hamiltonian of the entire system written in the basis $|j\rangle$ of atomic orbitals centered at a site $j$ is given by $\hat{H}$ $=\hat{H}_{\mathrm{NT}}+\hat{H}_{A}+\hat{H}_{B}+\hat{V}_{C}$, where $\hat{H}_{\mathrm{NT}}=\Sigma_{j, j^{\prime}}|j\rangle \gamma\left\langle j^{\prime}\right|$ is the Hamiltonian of the individual nanotube, $\hat{H}_{A}=|a\rangle \epsilon_{a}\langle a|$ and $\hat{H}_{B}$ $=|b\rangle \epsilon_{b}\langle b|$ are the Hamiltonians associated with the isolated atoms $A$ and $B$, respectively, and $\hat{V}_{C}=h\{|a\rangle\langle 0|+| 0\rangle\langle a|$ $+|b\rangle\langle n|+| n\rangle\langle b|\}$ refers to the coupling between the adatoms and the nanotube. The parameters $\gamma, \epsilon_{a}, \epsilon_{b}$, and $h$ are all matrices in spin and orbital spaces and correspond to the hopping between nearest-neighbor sites in the nanotube, the on-site potentials of atom $A$, of atom $B$, and the hopping between the nanotube atoms and the adatoms, respectively.
Likewise, the basis $|j\rangle$ represents vectors in the same linear space. It is evident from the expressions above that sites $j$ $=a$ and $j=b$ label the two adatoms and $j=0$ and $j=n$ label the nanotube sites that are coupled to the magnetic atoms.

Since we are interested in evaluating the energy required to rotate the magnetic moment of one adatom relatively to the other, it is instructive to start by expressing the total energy of the system in the FM configuration, i.e.,

$$
\mathcal{E}_{\mathrm{FM}}=\int_{-\infty}^{\infty} d \omega\left[\frac{\omega}{1+e^{\beta(\omega-\mu)}}\right]\left[\left(-\frac{1}{\pi}\right) \operatorname{Im} \operatorname{Tr} \sum_{j} G_{j, j}(\omega)\right],
$$

where $\beta=1 / k_{B} T, k_{B}$ is the Boltzmann constant, $T$ is the temperature, $\mu$ is the Fermi level, $G_{j, j}(\omega)$ is the Green function of an electron with energy $\omega$ localized at site $j$, and $\operatorname{Tr}$ stands for the trace over the orbital and spin degrees of freedom. To rotate the magnetic moment of one adatom, say atom $A$, we introduce a perturbation $V(\theta)$ localized on this atom that describes how the effective potential is altered by the rotation of an angle $\theta$. In terms of the Pauli matrices $\sigma_{x}, \sigma_{y}$, and $\sigma_{z}$, it is given by $V(\theta)=-V_{x} \otimes\left[(\cos \theta-1) \sigma_{z}+\sin \theta \sigma_{x}\right]$, where $V_{x}$ is a matrix in orbital space representing the strength of the local exchange potentials. The energy change that results from the perturbation $V(\theta)$ can be expressed by the respective changes produced in the Green function $G_{j, j}$ summed over all sites $j$ (including $j=a$ and $j=b$ ). Making use of suitable sum rules for Green functions, we can write this energy change as ${ }^{11,12}$

$$
\Delta \mathcal{E}(\theta)=\frac{1}{\pi} \int_{-\infty}^{\infty} d \omega\left[\frac{1}{1+e^{\beta(\omega-\mu)}}\right] \operatorname{Im} \operatorname{Tr} \ln \left[1-G_{a, a}(\omega) V(\theta)\right],
$$

where $G_{a, a}$ represents the Green function in the FM configuration projected at the atomic site $j=a$. Further manipulation of Eq. (2) leads to an explicit dependence of the integrand on the spin indices, i.e.,

$$
\begin{aligned}
\Delta \mathcal{E}(\theta)= & \frac{1}{\pi} \int_{-\infty}^{\infty} d \omega\left[\frac{1}{1+e^{\beta(\omega-\mu)}}\right] \\
& \times \operatorname{Im} \operatorname{Tr} \ln \left[1+2 V_{x}^{2}(1-\cos \theta) G_{a, b}^{\uparrow}(\omega) G_{b, a}^{\downarrow}(\omega)\right],
\end{aligned}
$$

where $G_{m, l}^{\sigma}(\omega)$ represents the propagator between sites $j=l$ and $j=m$ for electrons of spin $\sigma$ and energy $\omega$, and the trace is now only over orbital indices. Since the $\cos \theta$ in the integrand above is the only $\theta$-dependent term, it is reasonable to assume a Heisenberg-like angular dependence for $\Delta \mathcal{E}$ and conclude that the minimum energy is either at $\theta=0$ or $\theta=\pi$. By defining the coupling as $\Delta \mathcal{E}(\theta=\pi)$ we can infer not only about the strength of the indirect interaction between the adatoms but also whether their moments display a parallel or antiparallel alignment.

It is instructive to compare our technique with that based on the Ruderman-Kittel-Kasuya-Yosida (RKKY) theory, a method that has been widely used to study the indirect coupling between magnetic impurities within metallic hosts. 
Whereas the RKKY is a second-order perturbation theory, our method is nonperturbative, which means that the multiple-scattering terms that arise from the introduction of a perturbing potential are fully taken into account by the use of suitable sum rules in the Green functions. Our expression is therefore more general and is able to recover the RKKY results as a limiting case of small magnetic moments. In fact, this is what occurs if the logaritmic function in the integrand of Eq. (3) is expanded up to second order in powers of $V_{x}$.

For the sake of simplicity the electronic structure of the system will be treated here within the single-band tightbinding model. The expressions above are very general and by no means restricted to such a simple case. The results obtained here can be easily extended to a multiorbital description but bring no qualitative difference. Having transition-metal atoms in mind, the adatoms are described by a fivefold degenerate $d$ band with the appropriate occupation to represent typical magnetic materials. The carbon nanotube is also known to be well described by a single-band tightbinding model with suitable parameters that successfully reproduce the experimental data for the nanotube electronic structure. In this way, rather than matrices in orbital indices, all quantities in the integrand of Eq. (3) become scalar.

It is worth mentioning that the dependence of $\Delta \mathcal{E}$ on the distance between the two magnetic adatoms is contained entirely in the matrix elements $G_{a, b}^{\sigma}$ and $G_{b, a}^{\sigma}$. To make this dependence more explicit we can express the Green function $G$ associated with the system in the FM configuration in terms of another Green function associated with the separate parts (also in the FM configuration). In other words, by considering the coupling $h$ between the nanotube and the adatoms as a perturbation, we can use Dyson's equation to write the matrix elements of $G$ in terms of those associated with their isolated components. It is then straightforward to see that

$$
G_{b, a}^{\sigma}(\omega)=C\left(\frac{\mathcal{G}_{n, 0}}{1-P-Q \mathcal{G}_{n, 0} \times \mathcal{G}_{0, n}}\right),
$$

where $C=\mathcal{G}_{a, a} h^{2} \mathcal{G}_{b, b} /\left(1-\mathcal{G}_{a, a} h^{2} \mathcal{G}_{0,0}\right), \quad P=\mathcal{G}_{b, b} h^{2} \mathcal{G}_{n, n}$ and $Q$ $=\mathcal{G}_{b, b} h^{3} /\left(1-\mathcal{G}_{a, a} h^{2} \mathcal{G}_{0,0}\right)$. Since the Green function $\mathcal{G}$ represents the electronic propagator associated with the nanotube and adatoms in isolation, it is evident that $\mathcal{G}_{a, a}=1 /\left(\omega-\epsilon_{a}\right)$ and $\mathcal{G}_{b, b}=1 /\left(\omega-\epsilon_{b}\right)$. Regarding the Green functions $\mathcal{G}_{0, n}$ and $\mathcal{G}_{n, 0}$, these are the only matrix elements associated with impurity-free nanotubes that carry the spatial dependence of the indirect magnetic coupling between the adatoms. The indirect character of the coupling is evident in the expressions above when written in terms of the electronic propagators between the sites that are coupled to the magnetic adatoms. Put in another way, the presence of those off-diagonal propagators in the expression for the coupling indicates that the magnetic information between the moments is being carried back and forth by the conduction electrons of the nanotube host. Since these propagators play such an important role in determining the most energetically favorable magnetic configuration, in the following section we present a fully analytical method providing closed-form expressions for arbitrary matrix elements of the nanotube Green function, among which the elements $\mathcal{G}_{0, n}$ and $\mathcal{G}_{n, 0}$.

\section{GREEN FUNCTION OF CARBON NANOTUBES}

Carbon nanotubes are fullerenelike structures that can be regarded as graphene sheets wrapped up in cylindrical shape. The electronic structures of both graphene and carbon nanotubes are well described by a tight-binding model for the $\pi$ orbital, the only difference between them being the wave vector quantization along the circumferential direction of the tubes. With such a simple dispersion relation describing the band structure of pure tubes, one can obtain the corresponding single-particle Green functions without having to evaluate them numerically. In this section we derive an analytical closed-form expression for a general matrix element of the single-particle Green function of an infinitely long carbon nanotube. The propagator between any two sites of an impurity-free nanotube is obtained and will be used to calculate the indirect exchange coupling between two magnetic adatoms.

The tight-binding Hamiltonian for a carbon nanotube is diagonal when written in the basis $|\mathbf{k}, \pm\rangle$ defined as

$$
|\mathbf{k}, \pm\rangle=\sqrt{\frac{1}{2 L}} \sum_{l}\left[e^{i \vec{k} \cdot \vec{R}_{l}}|l, \bullet\rangle \pm e^{-i \phi(\vec{k})} e^{i \vec{k} \cdot \vec{R}_{l}^{o}}|l, \circ\rangle\right] .
$$

The basis $|l, \star\rangle$ represents atomic orbitals centered at a site located at $\vec{R}_{l}^{\star}$ that is labeled by a pair of indices $(l, \star)$, where $l$ is the two-atom cell index and $\star=\bullet$ or $\star=0$ represent the two inequivalent atomic sites of the hexagonal lattice. The quantity $L$ represents the total number of cells and the phase $\phi(\vec{k})$ in Eq. (5) is defined as $\phi(\vec{k})=\operatorname{Im} \ln \mathcal{H}$, where $\mathcal{H}=\gamma\left[e^{i k_{x} a \sqrt{3} / 3}+2 \cos \left(k_{y} a / 2\right) e^{-i k_{x} a \sqrt{3} / 3}\right]$. The corresponding eigenvalues $E_{ \pm}$are found due to the basis formed by two inequivalent carbon sites in the graphene structure. The band structure becomes one dimensional when quantization conditions on the wave vectors $k_{x}$ and/or $k_{y}$ are introduced. For achiral tubes, namely, armchair and zigzag, the wave vectors $k_{x}$ and $k_{y}$ are quantized, respectively. In this case, the nonquantized wave vector runs continuously along the first Brillouin zone, whereas the quantized counterpart is only allowed to assume discrete values. For chiral tubes in general, a linear combination of $k_{x}$ and $k_{y}$ imposes the quantization condition. The resulting one-dimensional energy dispersions for the armchair and zigzag nanotubes are given by ${ }^{13}$

$$
E_{ \pm}^{j}\left(k_{y}\right)= \pm \gamma \sqrt{1+4 \cos \left(\frac{j \pi}{M}\right) \cos \left(k_{y} \frac{a}{2}\right)+4 \cos ^{2}\left(k_{y} \frac{a}{2}\right)}
$$

and

$$
E_{ \pm}^{j}\left(k_{x}\right)= \pm \gamma \sqrt{1+4 \cos \left(\frac{\sqrt{3} k_{x} a}{2}\right) \cos \left(\frac{j \pi}{M}\right)+4 \cos ^{2}\left(\frac{j \pi}{M}\right)},
$$

respectively, where $M$ depends on the geometry of the tube and is directly related to its diameter. The integer $j$ 
$=1,2, \ldots, 2 M$ labels the different bands. The nearestneighbor electronic hopping is denoted by $\gamma$ and is hereafter considered to be our energy unit. Notice that the bands are symetrically distributed around $E=0$.

In what follows we calculate an arbitrary matrix element of the Green function associated with both armchair and zigzag nanotubes, whose band structures are given by Eqs. (6) and (7). Green functions for chiral tubes are also possible to be obtained within the same formalism, but the corresponding unit cells can be very large. The general formula for the single-electron Green function is

$$
\mathcal{G}(\omega)=\sum_{\vec{k}}\left[\frac{|\vec{k},+\rangle\langle\vec{k},+|}{\omega-E_{+}(\vec{k})}+\frac{|\vec{k},-\rangle\langle\vec{k},-|}{\omega-E_{-}(\vec{k})}\right],
$$

where $\omega$ is the energy. Introducing Eq. (5) into Eq. (8), we obtain an expression for the Green function between any two sites $|j, \star\rangle$ and $\left|j^{\prime}, \star^{\prime}\right\rangle$ of a graphene sheet. For simplicity, we consider the propagation between two equivalent sites in different cells. In this case the matrix element of the Green function is given by

$$
\left\langle l, \star|\mathcal{G}(\omega)| l^{\prime}, \star\right\rangle=\frac{1}{2 L} \sum_{\vec{k}}\left[\frac{e^{i \vec{k} \cdot\left(\vec{R}_{l}^{\star}-\vec{R}_{l^{\prime}}^{\star}\right)}}{\omega-E_{+}(\vec{k})}+\frac{e^{i \vec{k} \cdot\left(\vec{R}_{l}^{\star}-\vec{R}_{l^{\prime}}^{\star}\right)}}{\omega-E_{-}(\vec{k})}\right] .
$$

\section{A. Armchair nanotubes}

The sum over $\vec{k}$ in Eq. (9) can be transformed into an integral over $k_{y}$. The wave vector $k_{x}$ remains discrete and its values are $k_{x}=(j / M)(2 \pi / \sqrt{3} a)$. In addition, by taking into account that $E_{-}(\vec{k})=-E_{+}(\vec{k})$, the expression above is rewritten as

$$
\mathcal{G}_{l, l^{\prime}}(\omega)=\frac{a}{4 M \pi} \sum_{k_{x}} \int_{-\pi / a}^{\pi / a} d k_{y}\left[\frac{\omega e^{i \vec{k} \cdot\left(\vec{R}_{l}^{\cdot}-\vec{R}_{l^{\prime}}\right)}}{\omega^{2}-E_{+}^{2}\left(k_{x}, k_{y}\right)}\right],
$$

For simplicity, in Eq. (10) we have dropped the bra and ket representing the initial and ending sites. For each value of $k_{x}$ we have a corresponding one-dimensional band structure. The integral above can be evaluated by extending $k_{y}$ to the complex plane and changing the integration contour from a straight line on the real axis to the boundaries of a semiinfinite rectangle in the upper half-plane whose base lies on the real axis between $-\pi / a$ and $\pi / a$. By determining the poles and their respective residues we can evaluate the integral for each band index $k_{x}$. The poles, labeled $q_{j}^{ \pm}$, are solutions of the following equation:

$$
\cos \left(q_{j}^{ \pm} \frac{a}{2}\right)=-\frac{1}{2}\left\{\cos \left(\frac{j}{M} \pi\right) \pm \sqrt{\frac{\omega^{2}}{\gamma^{2}}-\sin ^{2}\left(\frac{j}{M} \pi\right)}\right\},
$$

and the corresponding residues are

$$
\operatorname{Res}\left[q_{j}^{ \pm}\right]=\frac{\omega e^{i K_{j}^{ \pm} \cdot\left(\vec{R}_{l}-\vec{R}_{l^{\prime}}\right)}}{4 \pi M t^{2}\left\{\cos \left(\frac{j}{M} \pi\right) \sin \left(q_{j}^{ \pm} \frac{a}{2}\right)+2 \cos \left(q_{j}^{ \pm} \frac{a}{2}\right) \sin \left(q_{j}^{ \pm} \frac{a}{2}\right)\right\}},
$$

where $\vec{K}_{j}^{ \pm}=(j 2 \pi / \sqrt{3} M a) \vec{e}_{x}+\left(q_{j}^{ \pm}\right) \vec{e}_{y}$. Equation (11) does not define the poles $q_{j}^{ \pm}$uniquely. By defining the cosine of pole $q_{j}^{ \pm}$but not its sine, the residue in Eq. (12) is free to assume two distinct values. The residue depends on the correct sign of $\sin \left[q_{j}^{ \pm}(a / 2)\right]$, which is obtained by imposing that the imaginary part of diagonal elements of retarded Green functions must be negative. The expression for $\mathcal{G}_{l, l^{\prime}}(\omega)$ then becomes

$$
\mathcal{G}_{l, l^{\prime}}(\omega)=\sum_{j} \frac{i \omega e^{i \vec{K}_{j}^{ \pm} \cdot\left(\vec{R}_{l}^{\star}-\vec{R}_{l^{\prime}}^{\star}\right)}}{M t^{2}\left\{\cos \left(\frac{j}{M} \pi\right) \sin \left(q_{j}^{ \pm} \frac{a}{2}\right)+2 \cos \left(q_{j}^{ \pm} \frac{a}{2}\right) \sin \left(q_{j}^{ \pm} \frac{a}{2}\right)\right\}} .
$$

This expression gives the matrix elements for the Green function of an armchair nanotube. Although the derivation of Eq. (13) assumed equivalent sites in distinct cells, the expression is almost the same for inequivalent sites, except for a multiplying phase factor $e^{-i \phi\left(K^{ \pm}\right)}$.

It is interesting to look at the physical significance of those poles. Each value of $k_{x}$ yields a corresponding $k_{y}$ and since they are obtained from the band structure for a given energy $\omega$, they are just the coordinates of constant-energy surface along the axial direction. In other words, they indicate the wave vector $q_{j}^{ \pm}$with which electrons of energy $\omega$ propagate along the axial direction of the nanotube. Many discrete values of $k_{x}$ have no real $k_{y}$ components, and therefore $q_{j}^{ \pm}$assumes imaginary values leading to evanescent contributions to the Green functions.

\section{B. Zigzag nanotubes}

In the case of zigzag tubes the $k_{y}$ components are quantized whereas the $k_{x}$ run continuously over the Brillouin 
zone. The expression for the Green function becomes

$$
\mathcal{G}_{l, l^{\prime}}(\omega)=\frac{a \sqrt{3}}{2 M \pi} \sum_{k_{y}} \int_{-\pi / a \sqrt{3}}^{\pi / a \sqrt{3}} d k_{x}\left[\frac{\omega e^{i \vec{k} \cdot\left(\vec{R}_{l}^{-}-\vec{R}_{l^{\prime}}\right)}}{\omega^{2}-E_{+}^{2}\left(k_{x}, k_{y}\right)}\right] .
$$

Analogously to the armchair case, the integral above can be evaluated by indentifying the poles and summing over the respective residues. The poles, labeled $q_{j}$, are obtained through the following equation:

$$
\cos \left(q_{j} \frac{a \sqrt{3}}{2}\right)=\frac{\frac{\omega^{2}}{\gamma^{2}}-1-4 \cos ^{2}\left(\frac{\pi j}{M}\right)}{4 \cos \left(\frac{\pi j}{M}\right)}
$$

and the corresponding residues are

$$
\operatorname{Res}\left[q_{j}\right]=\frac{\omega e^{i \vec{K}_{j} \cdot\left(\vec{R}_{l}^{*}-\vec{R}_{l^{\prime}}\right)}}{4 \pi M t^{2} \cos \left(\frac{\pi j}{M}\right) \sin \left(q_{j} \frac{a \sqrt{3}}{2}\right)},
$$

where $\vec{K}_{j}=q_{j} \vec{e}_{x}+(2 \pi j / M a) \vec{e}_{y}$. Finally, the expression for $\mathcal{G}_{l, l^{\prime}}(\omega)$ is given by

$$
\mathcal{G}_{l, l^{\prime}}(\omega)=\sum_{j} \frac{i \omega e^{i \hat{K}_{j}^{ \pm} \cdot\left(\vec{R}_{\left.l^{*}-\vec{R}_{l^{\prime}}\right)}\right)}}{M t^{2}\left\{\cos \left(\frac{j}{M} \pi\right) \sin \left(\sqrt{3} q_{j}^{ \pm} \frac{a}{2}\right)\right\}} .
$$

This expression gives the matrix elements for the Green function of a zigzag nanotube.

Despite having presented closed-form analytical expressions for the Green functions of armchair and zigzag nanotubes separately, common features in the expressions must be highlighted. The energy dependence is not only given by the energy on the numerator of Eqs. (13) and (17), but it is implicitly contained in the poles, as shown in Eqs. (11) and (15). Likewise, the $M$ dependence is also implicit in the poles. Each term of the summation corresponds to a band contribution to the single particle Green function. One major advantage of an expression for the Green function that does not depend on numerical evaluation, is that it allows a transparent analysis of the relationship between the electronic structure and the relevant parameters involved. For diagonal elements of the Green functions, $j=j^{\prime}$ vanishes the argument of the exponential in the numerator of Eqs. (13) and (17) and the imaginary part gives a direct expression for the density of states for both armchair and zigzag nanotubes.

\section{RESULTS AND DISCUSSION}

The indirect magnetic coupling can now be calculated by inserting the required matrix elements of $\mathcal{G}$ into Eq. (4) and evaluating the corresponding energy integral of Eq. (3). By changing the relative positions between the adatoms we can see how the coupling depends on their separation. Furthermore, by changing the nature of the adatoms we can also investigate how the coupling responds at the presence of different magnetic entities. We can also investigate how the coupling behaves for different types and sizes of nanotubes and finally, how it changes with temperature.

\section{A. Zigzag (metallic)}

We start by considering a metallic zigzag nanotube defined by the indices $(6,0)$. The solid line of Fig. 2 shows the coupling $\Delta \mathcal{E}(\pi)$ as a function of the distance $D$ between adatoms along the axial direction of the tube. The parameters were chosen to reproduce the adatom occupation of Co atoms. The first noticeable feature in this result is the monotonicity of the coupling. As a general physical principle, it is well known that the indirect coupling between magnetic impurities embedded in a metallic host oscillates as the impurities are moved further apart. ${ }^{9}$ At first glance, one may think that this principle is not applicable to carbon nanotubes. However, as we show below, this is not the case, the lack of oscillations being the result of a commensurability effect.

On a three-dimensional system, in an analogy to the de Haas van Alphen effect in metals, the oscillatory behavior of the coupling is determined by the topology of the bulk Fermi surface (FS) of the host material. ${ }^{14}$ More precisely, each FS extremum along a specific direction contributes with one oscillatory component of a definite period. This period is determined by the caliper associated with that extremum. For a CN, the Fermi "surface" is actually a discrete set of points. Inspecting the zigzag's Fermi points one sees that there is only one extremum, thus the coupling should oscillate with one single period. Nevertheless, the solid line in Fig. 2 is always negative indicating a preferential antiferromagnetic alignment between the adatom moments. For infinitely large separations, the coupling should vanish but it does so without sign changes or any oscillations. This is because the period coming from the caliper associated with that single extremum is actually commensurate with the nearest-neighbor distance in the axial direction. In other words, the expected oscillations are hidden by the fact that the coupling is being probed at intervals that are of the same length as the unit cell

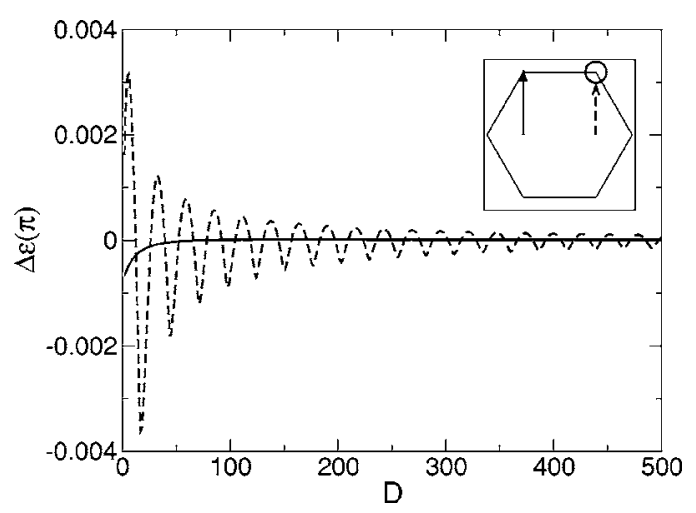

FIG. 2. Exchange coupling between Co adatoms as a function of the adatom separation on a zigzag $(6,0)$ nanotube (solid line). The dashed line is the coupling when the nanotube is slightly doped, corresponding here to a small shift in its Fermi energy. The inset shows schematically the Fermi wave vector for pure (solid arrow) and doped (dashed arrow) tubes. All coupling values are given in units of the nearest-neighbor electronic hopping $\gamma$. 
separation. To prove this point, we introduce a slight shift in the Fermi level that would correspond to either doping the nanotube or to the action of a gate voltage affecting the occupation of the system. In this case, the Fermi surface of the nanotube no longer consists of isolated points at the corners of the Brillouin zone. The points in reciprocal space are now located at the intersection between small circularlike curves centered at the hexagonal corners of the Brillouin zone and the discrete quantization lines along the $k_{y}$ direction, as shown in the inset of Fig. 2. The inset displays the wave vectors that contribute to the oscillatory coupling, which clearly shows that they no longer coincide with the dimensions of the Brillouin zone. A small shift in the Fermi level $\left(\Delta E_{F}=0.06 \gamma\right.$ in this case) is sufficient to destroy the commensurability effect in the coupling, as shown by the longperiod oscillatory curve (dashed line) of Fig. 2.

The lack of oscillations in the indirect coupling is sufficiently robust to appear with other magnetic adatoms. In fact, by changing the parameters such that the $d$-band occupation of the adatoms corresponds to that of $\mathrm{Ni}$ atoms, we find a similar behavior for the coupling, the only difference being the overall sign. This is illustrated by Fig. 3 in which the full line depicts the calculated coupling between two $\mathrm{Ni}$ adatoms as a function of their longitudinal separation. Rather than an antiferromagnetic alignment between the moments, in this case we find that they tend to align in a ferromagnetic fashion. Likewise, the monotonicity of the coupling remains unnaffected by varying the tube diameter. This is confirmed by the dashed and dot-dashed lines in Fig. 3 in which the coupling between Ni adatoms is plotted as a function of their separation for a $(9,0)$ and a $(12,0)$ tube, respectively. The inset also shows that the coupling decays with the tube diameter as $1 / R$, where $R$ is the nanotube radius. This is hardly surprising, bearing in mind that the density of electronic states of a nanotube has a similar behavior and that the coupling is the result of an indirect coupling mediated by the nanotube electrons.

\section{B. Zigzag (semiconductor)}

For the case of semiconducting tubes, the lack of extended states at the Fermi level supresses the long-range character of the indirect coupling, even in one dimension. In this case the coupling decays exponentially with the adatom separation, as shown by the full line in Fig. 4 for a $(31,0)$ nanotube. The choice of such a large diameter tube is justified to illustrate the fact that a minute change in the occupation is capable of moving the Fermi level into the zone of extended states and producing a remarkable effect in the coupling. The dashed line shows the coupling for a slightly doped nanotube $\left(\Delta E_{F}=0.06 \gamma\right)$ displaying similar behavior to the metallic case, where a long-period long-ranged oscillatory coupling is observed. From a virtually uncoupled configuration, the coupling between adatom moments can be switched on by changing the occupation of the nanotube. Consequently, by using gate voltages to control the Fermi level of the system, in principle one can reversibly turn magnetically uncoupled adatoms into fully aligned magnetic moments, a promising feature for use in spintronic devices.

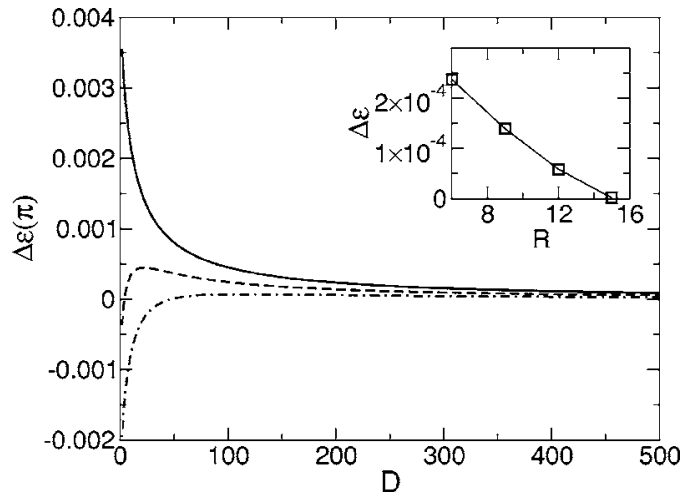

FIG. 3. Exchange coupling as a function of longitudinal separation $D$ between Ni adatoms on $(6,0)$ (solid line), (9,0) (dashed line), and $(12,0)$ (dot-dashed line) nanotubes. The inset shows how the coupling amplitude decays as a function of tube diameter. All coupling values are given in units of the nearest-neighbor electronic hopping $\gamma$.

\section{Armchair}

For the armchair tubes, there are two contributory wave vectors extracted from their Fermi surface, and as such there should be two oscillatory components in the exchange coupling. These are identified in the inset of Fig. 5(a) together with the coupling as a function of the adatom separation in the main graph. Once again, one of the periods is commensurate with the interatomic distance, which makes the coupling to oscillate with a noncommensurate period superimposed to a steady decay. The coupling at finite temperature [shown in Fig. 5(b)] tends to decay faster, which can be explained by the fact that the thermal energy partially suppresses the propagators between adatoms, thus reducing the efficiency with which the electronic carriers transport the magnetic information back and forth.

Concerning the rate of decay of the coupling as a function of the adatom separation, all examples above decay slowly in the asymptotic limit $(D \gg 1)$. It is known that in onedimensional structures the coupling decays approximately as $1 / D$ for $D \gg 1$. This is what we observe approximately when

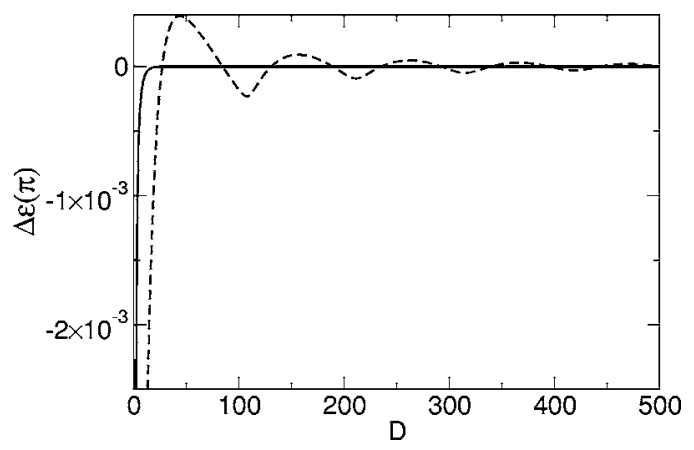

FIG. 4. Exponentially decaying exchange coupling between Co adatoms on a semiconducting $(31,0)$ nanotube (solid line). A small amount of doping is enough to populate the tube's conduction band and give origin to an oscillating, long-ranged exchange coupling (dashed line). All coupling values are given in units of the nearestneighbor electronic hopping $\gamma$. 
(a)

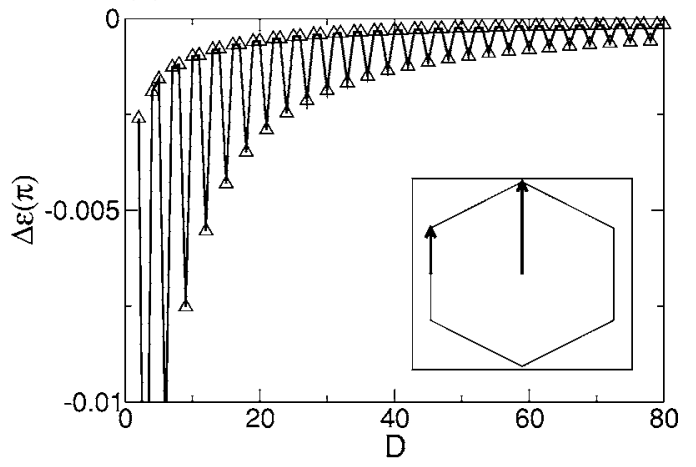

(b)

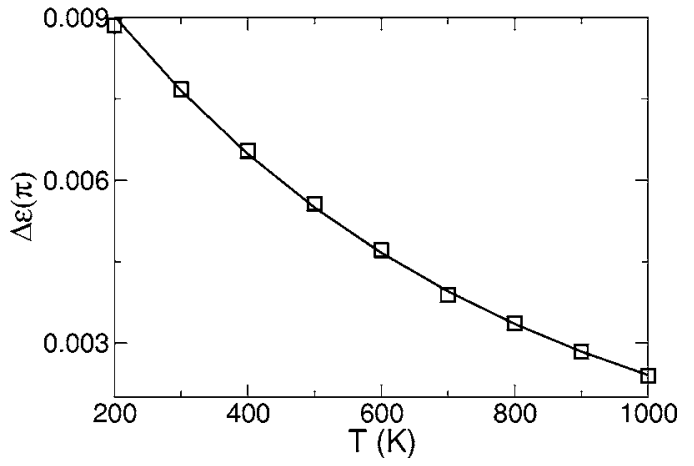

FIG. 5. (a) Exchange coupling as a function of longitudinal separation $D$ between two Co adatoms on a $(6,6)$ armchair nanotube. The inset shows the wave vectors contributing to the coupling with different oscillation periods. (b) Amplitude of the exchange coupling as a function of temperature. All coupling values are given in units of the nearest-neighbor electronic hopping $\gamma$.

impurities embedded in the nanotube are considered, instead of adatoms. As the tube diameter increases, the system approaches a two-dimensional-like structure and the coupling tends to decay a little faster. This is displayed in Fig. 6 showing the coupling for armchair nanotubes of different diameters. The coupling between adatoms has a more complicated asymptotic behavior. For most of the range of $D$ values we investigated it was not possible to identify a unique decay rate. In the regions where the decay rate is well-defined it may be even slower than $1 / D$. Differences between the coupling decay rates for adatoms and embedded impurities have been reported in other systems. ${ }^{15}$ It is worth mentioning that such a slow decaying rate in the coupling leads to a longranged magnetic interaction that, when combined with the lack of oscillations introduced by the commensurability effect, can be potentially used to induce ordered magnetic structures on the surface of a carbon nanotube.

\section{CONCLUSIONS}

In summary, we have presented expressions for the indirect exchange coupling between magnetic adatoms mediated by the conduction electrons of carbon nanotubes. It is obvious from the expressions that the coupling, written in terms of a few matrix elements of the single-particle Green functions, depends on the electronic carriers transporting the

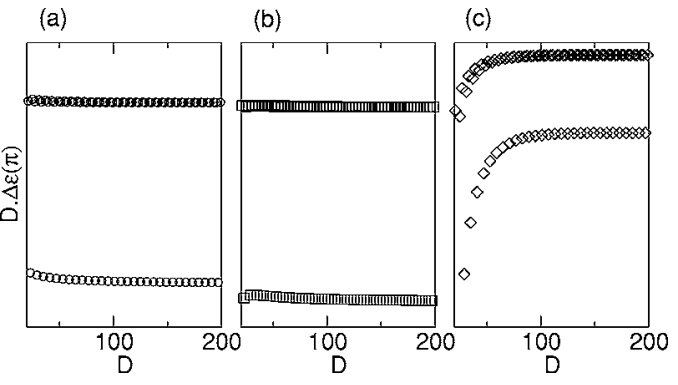

FIG. 6. Exchange coupling $\Delta \mathcal{E}(\pi)$ multiplied by the distance between impurities $D$, as a function of $D$, for (a) $(6,6)$, (b) $(24,24)$, and (c) $(48,48)$ armchair nanotubes. It is evident that in the $(6,6)$ and $(24,24)$ nanotubes the coupling decays as $1 / D$, whereas in the $(48,48)$ tube it decays faster. All coupling values are given in units of the nearest-neighbor electronic hopping $\gamma$.

magnetic information back and forth to both adatoms. We show that, contrary to expectations, we find a monotonic behavior in the coupling between magnetic adatoms adhered to the surface of metallic zigzag nanotubes. Rather than an intrinsic property, we have shown that the lack of usual oscillations results from a commensurability effect. In other words, the oscillations are hidden by the fact that the contributory wave vectors have the same size as the Brillouin zone, forcing the periods to coincide with the atomic spacing. In this commensurate regime the coupling does not change sign but decays monotonically. The overall sign of this monotonic coupling depends on the nature of the adatoms attached to the nanotube. In the case of positive coupling, a FM alignment between the moments persists as we move the adatoms apart, where the coupling magnitude decays rather slowly as approximately $1 / D$. Such a long-range interaction may be responsible for inducing magnetic order in the nanotube.

The coupling across semiconducting tubes is exponentially damped, which results from the lack of extended states to transport the magnetic information between the adatoms. By moving the occupation into the conduction band we show a dramatic change in the coupling, departing from a configuration with random alignment between magnetic moments into another that is highly ordered due to the predominantly positive long-ranged long-period oscillatory coupling. We argue that a controlled gate voltage can potentially tune the occupation in such a way that the magnetic interaction can be switched on and off in a reversible fashion.

Oscillatory coupling is found on armchair metallic tubes. In this case, two periods are identified, one commensurate and another incommensurate with the lattice. The resulting coupling consists of a monotonically decaying contribution superimposed to a $1 / D$-modulated oscillatory coupling. Concerning the dependence of the coupling on other parameters, its diameter dependence follows the $1 / R$ behavior displayed by the density of states. As expected, the coupling decays with temperature but not as fast as the coupling between moments embedded in three-dimensional structures.

Finally, it is worth emphasizing that by controlling the alignment of magnetic structures attached to the surface of carbon nanotubes one can potentially select the way in which the systems will respond to magnetic excitations. Moreover, 
bearing in mind that the transport properties of magnetically doped structures are sensitive to the alignment of their magnetizations, the understanding of how separate moments are coupled across nanotubes could be a major step forward in the search for and control of spin-valve effects in those structures.

\section{ACKNOWLEDGMENTS}

Useful discussions with R. B. Muniz are gratefully acknowledged. The authors thank Science Foundation Ireland for financial support. A.T.C., Jr. is grateful to the Brazilian agencies CNPq, FAPEMIG, and FINEP for financial support.
*Electronic address: antc@stout.ufla.br

†Electronic address: ferreirm@tcd.ie

${ }^{1}$ K. Tsukagoshi, B. W. Alphenaar, and H. Ago, Nature (London) 401, 572 (1999).

${ }^{2}$ M. S. Ferreira and S. Sanvito, Phys. Rev. B 69, 035407 (2004).

${ }^{3}$ O. Céspedes, M. S. Ferreira, S. Sanvito, M. Kociak, and J. M. D. Coey, J. Phys.: Condens. Matter 16, 155 (2004).

${ }^{4}$ S. B. Fagan, R. Mota, R. J. Baierle, A. J. R. da Silva, and A. Fazzio, Mater. Charact. 50, 183 (2003).

${ }^{5}$ S. B. Fagan, R. Mota, A. J. R. da Silva, and A. Fazzio, Phys. Rev. B 67, 205414 (2003).

${ }^{6}$ S. B. Fagan, R. Mota, A. J. R. da Silva, and A. Fazzio, Physica B 340, 982 (2003).

${ }^{7}$ C. K. Yang, J. Zhao, and J. P. Lu, Phys. Rev. Lett. 90, 257203 (2003).
${ }^{8}$ D. Altbir, P. Vargas, and J. d'Albuquerque e Castro, Phys. Rev. B 64, 012410 (2001).

${ }^{9}$ M. D. Stiles, J. Magn. Magn. Mater. 200, 322 (1999).

${ }^{10}$ U. Larsen, J. Phys. D 15, 101 (1985).

${ }^{11}$ J. d'Albuquerque e Castro, M. S. Ferreira and R. B. Muniz, Phys. Rev. B 49, R16062 (1994).

${ }^{12}$ The difference to the original expression for the indirect coupling derived in Ref. 11 is the absence of in-plane translational invariance that exists in the case of multilayered structures.

${ }^{13}$ R. Saito, G. Dresselhaus and M. S. Dresselhaus, Physical Properties of Carbon Nanotubes (World Scientific, Singapore, 1998).

${ }^{14}$ D. M. Edwards, J. Mathon, R. B. Muniz, and M. S. Phan, Phys. Rev. Lett. 67, 493 (1991).

${ }^{15} \mathrm{~S}$. Frota-Pessoa (private communication). 